\title{
Activation of the miR-34a-Mediated SIRT1/mTOR Signaling Pathway by Urolithin A Attenuates D-Galactose-Induced Brain Aging in Mice
}

\author{
Peng Chen ${ }^{1} \cdot$ Fuchao Chen $^{2} \cdot$ Jiexin $\mathrm{Lei}^{3} \cdot{\text { Qiaoling } \mathrm{Li}^{4} \cdot \text { Benhong Zhou }}^{1}$ (D)
}

Published online: 1 July 2019

(C) The American Society for Experimental NeuroTherapeutics, Inc. 2019

\begin{abstract}
Despite tremendous advances in modern medicine, effective prevention or therapeutic strategies for age-related neurodegenerative diseases such as Alzheimer's disease (AD) remain limited. Currently, accumulating evidence has demonstrated that microRNAs (miRNAs) are increasingly associated with age-related diseases and are emerging as promising therapeutic targets. Urolithin A, a metabolite compound resulting from the transformation of ellagitannins by gut bacteria, has been reported to have anti-oxidant, anti-inflammatory, and anti-apoptotic properties. The present study primarily focused on the ameliorative effect of urolithin A on aging mice and on the exploration of the potential mechanisms of such an ameliorative effect on cognitive impairment and brain aging. In this study, we first tested the neuroprotective effect of urolithin A using an in vitro $\mathrm{H}_{2} \mathrm{O}_{2}$-induced PC12 cell oxidative damage model. The in vivo D-gal-induced brain aging model showed that urolithin A significantly suppressed the upregulation of miR-34a induced by D-gal. Moreover, target genes associated with miR-34a were also examined. Urolithin A supplementation ameliorated apoptosis induced by D-gal and rescued miR-34a overexpression-induced impaired autophagy in brain aging mice after a 2-month administration. Furthermore, urolithin A activated autophagy by upregulating the SIRT1 signaling pathway and downregulating the mTOR signaling pathway. In conclusion, urolithin A may exert neuroprotective effects and may aid in preventing D-gal-induced brain aging through activation of the miR-34a-mediated SIRT1/mTOR signaling pathway.
\end{abstract}

Key Words Urolithin A $\cdot$ D-Gal $\cdot$ aging $\cdot$ miR-34a $\cdot$ autophagy $\cdot$ SIRT1/mTOR signal pathway

\section{Introduction}

Alzheimer's disease $(\mathrm{AD})$ is one of the most common agingassociated neurodegenerative diseases in the elderly population worldwide and is characterized by a gradual and incremental loss of cognitive and motor performances [1,2]. Although specific pathological hallmarks of $\mathrm{AD}$ have been identified and included

Peng Chen and Fuchao Chen contributed equally to this work.

Benhong Zhou

benhongzh@whu.edu.cn

1 Department of Pharmacy, Renmin Hospital of Wuhan University, Wuhan 430060, Hubei, People's Republic of China

2 Department of Pharmacy, Dongfeng Hospital, Hubei University of Medicine, Shiyan 442008, Hubei, People's Republic of China

3 Department of Endocrinology, Renmin Hospital of Wuhan University, Wuhan 430060, Hubei, People's Republic of China

4 School of Pharmaceutical Sciences, Wuhan University, Wuhan 430071, Hubei, People's Republic of China amyloid precursor protein (APP), tau, and beta-site APP-cleaving enzyme 1 (BACE1), the exact biochemical mechanism of $\mathrm{AD}$ is still not fully known, and effective treatments still remain scarce $[3,4]$. Up to now, noncoding RNAs have been paid great attention and interest of the researchers in recent years as a potentially new and crucial layer of biological regulation [5].

MicroRNAs (miRNAs) constitute a recently discovered class of noncoding RNAs (ncRNAs, 18-25 nucleotides in length) that play key roles in the regulation of mRNA stability and gene expression [6]. It is well known that miRNAs are abundant in the brain and play a crucial role in neurodevelopment, synaptic plasticity, and pathological mechanisms for neurodegenerative diseases $[7,8]$. Accumulating evidence suggests that alterations in miRNA expression could be involved in the pathogenesis of $\mathrm{AD}$ [9]. It has been confirmed that miR-34a is a key factor in the induction of senescence, cell cycle arrest, and apoptosis, which is closely related to a variety of aging-associated diseases including $\mathrm{AD}$ [10]. Therefore, miRNAs may be promising therapeutic targets for pharmacological intervention in the treatment of neurodegenerative disorders. 
To date, natural metabolic active products have been widely tested as important sources for drug discovery in anti-aging and aging-related diseases. Urolithin $\mathrm{A}\left(\mathrm{C}_{13} \mathrm{H}_{8} \mathrm{O}_{4}, 3,8\right.$ dihydroxyl-6H-dibenzo [b,d] pyrane-6-ketone; the chemical structure is shown in Fig. S1A) is a major metabolite produced by rats and humans after the consumption of pomegranate juice or pure ellagitannin geraniin [11]. Pharmaceutical studies on urolithin A have reported that it possesses many pharmacological functions such as anti-oxidative, anti-inflammatory, anti-apoptotic, and cardioprotective functions [12]. Recently, the anti-aging effect of urolithin A has drawn considerable attention, and it has been confirmed that urolithin A can induce mitophagy, prolong the lifespan of C. elegans, and increase muscle function in rodents [13]. However, there are still no scientific reports to date on the protective effect of urolithin A on D-galactose (D-gal)-induced aging and degenerative brain disease.

It has been reported that mammalian target of rapamycin (mTOR) and silent mating type information regulation 2 homologue 1 (sirtuin 1, SIRT1) are involved in the regulation of aging through the modulation of the functional status of autophagy [14]. More importantly, accumulating data have revealed that miRNAs can be regulated by dietary and pharmacological agents [15]. Thus, we propose the hypothesis that a potential approach for the prevention and treatment of agingassociated diseases could be accomplished by inducing autophagy via the miR-34a-mediated SIRT1/mTOR signaling pathway. The aim of the present study was therefore to estimate the effect of urolithin A on D-gal-induced aging mice with cognitive deficits and brain injury and to validate our hypothesis by evaluating protein and gene expression associated with the miR-34a/SIRT1/mTOR signaling pathway, which will provide a comprehensive foundation for the theoretical basis and practical application of urolithin $\mathrm{A}$ in the treatment of aging-associated diseases.

\section{Materials and Methods}

\section{Chemicals and Regents}

D-Galactose (D-gal, lot no. 1602032) was purchased from Guangyuan Hengxin Technology Development Co., Ltd. (Beijing, China, purity > 99\%). Urolithin A standard was purchased from Sigma Chemical Co. (St. Louis, MO, lot no. 100151-231, purity $>99 \%$ ). Commercial kits for measuring malondialdehyde (MDA, no. 20180924), monoamine oxidase (MAO, no. 20180951), superoxide dismutase (SOD, no. 20181042), total anti-oxidant capacity (T-AOC, no. 20180516), glutathione peroxidase (GSH-Px, no. 20180614), catalase (CAT, no. 20180512), and acetylcholinesterase (AChE, no. 20180741) were purchased from Nanjing Jiancheng Bioengineering Institute (Nanjing, China). ELISA assay kits for tumor necrosis factor- $\alpha$ (TNF- $\alpha$, no. 20180413), interleukin-1 beta (IL-1 $\beta$, no. 20180520), and interleukin-6 (IL-6, no. 20180701) were purchased from Santa Cruz Biotechnology (Santa Cruz, CA). Cleaved caspase-3, Bcl-2, Bax, LC-3, p62, Atg7, SIRT1, phospho-p53, p53, p21, GFAP, and mTOR antibodies were obtained from Thermo Fisher Scientific (Shanghai, China). All other solvents and chemicals used in the study were of analytical grade.

\section{Neuroprotective Effects of Urolithin A on $\mathrm{H}_{2} \mathrm{O}_{2}$-Induced Cytotoxicity}

\section{Cell Culture}

PC12 cells, a rat cell line derived from pheochromocytoma cells, were obtained from the China Center for Type Culture Collection and cultured in minimum essential medium (MEM) supplemented with $10 \%$ fetal bovine serum, 100 units $\mathrm{mL}^{-1}$ penicillin, and $100 \mu \mathrm{g} \mathrm{mL}^{-1}$ streptomycin sulfate at $37{ }^{\circ} \mathrm{C}$ with $5 \% \mathrm{CO}_{2}$. Cell viability was estimated by the CCK (WST-8) assay. Cells were seeded in 96-well culture plates $\left(1 \times 10^{3}\right.$ cells/well $)$. Prior to exposure to $100 \mu \mathrm{M} \mathrm{H}_{2} \mathrm{O}_{2}$ for $2 \mathrm{~h}, \mathrm{PC} 12$ cells were pretreated with urolithin A $(50 \mu \mathrm{g} / \mathrm{mL}, 30 \mu \mathrm{g} / \mathrm{mL}$, and $10 \mu \mathrm{g} / \mathrm{mL})$ for $24 \mathrm{~h}$. Ten microliters of CCK solution was added to each well, and the mixtures were incubated for an additional $1 \mathrm{~h}$ at $37^{\circ} \mathrm{C}$. Finally, the cells were dissolved in $100 \mathrm{~mL}$ of DMSO, and absorbance at $450 \mathrm{~nm}$ was measured in a Victor 1420 microplate reader (Perkin Elmer, Waltham, MA).

Cell cytotoxicity was measured with the lactate dehydrogenase (LDH) release assay. Neuronal PC12 cells were treated with $\mathrm{H}_{2} \mathrm{O}_{2}$ and incubated at $37{ }^{\circ} \mathrm{C}$ for $2 \mathrm{~h}$ in 96 -well plates. Then, urolithin A $(50 \mu \mathrm{g} / \mathrm{mL}, 30 \mu \mathrm{g} / \mathrm{mL}$, and $10 \mu \mathrm{g} / \mathrm{mL})$ was added to the culture $24 \mathrm{~h}$ prior to $\mathrm{H}_{2} \mathrm{O}_{2}$ addition, and the $\mathrm{LDH}$ content was determined using the LDH assay. Briefly, the reactions were conducted in a 96-well plate containing a $30-\mu \mathrm{L}$ aliquot of the medium and $60-\mu \mathrm{L}$ kit reagent and were incubated for $30 \mathrm{~min}$ at room temperature. Finally, the absorbance of each well was measured at $490 \mathrm{~nm}$ using a microplate reader.

\section{Assessment of Apoptosis by Flow Cytometry}

To further verify the effect of urolithin $\mathrm{A}$ on $\mathrm{H}_{2} \mathrm{O}_{2}$-induced apoptosis in PC12 cells, an Annexin V-FITC apoptosis detection kit was used. After treatment with urolithin $\mathrm{A}(50 \mu \mathrm{g} / \mathrm{mL}$, $30 \mu \mathrm{g} / \mathrm{mL}$, and $10 \mu \mathrm{g} / \mathrm{mL}$ ) for $24 \mathrm{~h}$, cells were collected by trypsin treatment and then washed twice with PBS. Then, $500 \mu \mathrm{L}$ binding buffer was added to resuspend the cells, and $5 \mu \mathrm{L}$ fluorescein-conjugated Annexin V and $5 \mu \mathrm{L}$ PI were added to each tube and incubated for $15 \mathrm{~min}$. Finally, apoptosis was detected by flow cytometry. 


\section{Experimental Animals and Model}

Male Institute of Cancer Research (ICR) mice (aged 46 weeks, body weight from 18-22 g) were purchased from Wuhan Institute of Biological Products Co., Ltd. (Wuhan, China). The animals used in this work were housed in individual standard cages and were kept on a 12-h light-dark cycle in a temperature-controlled room at 21 to $23{ }^{\circ} \mathrm{C}$, with free access to water and food. All procedures were approved by the internal ethical committee of Wuhan University (IACUCWU protocol number: SCXK (E) 2013-0004).

After 7 days of adaptive feeding, 60 mice were randomly divided into 5 groups, with 12 mice per group, named the normal control group, aging model group (D-gal), high-dose urolithin Atreated group $(150 \mathrm{mg} / \mathrm{kg})$, medium-dose urolithin A-treated group (100 $\mathrm{mg} / \mathrm{kg}$ ), and low-dose urolithin A-treated group $(50 \mathrm{mg} / \mathrm{kg}$ ). Except for the normal control group, all groups received daily subcutaneous injection (SI) of D-gal, which was dissolved in $0.9 \%$ normal saline (NS) at a dose of $150 \mathrm{mg} / \mathrm{kg} /$ day for 8 weeks $[15,16]$. Then, mice in the urolithin A group were subcutaneously and orally gavaged, whereas the mice in the control group were injected with equal volumes of $0.8 \%$ Tween 80 and concomitantly administered normal saline by gavage [17]. Furthermore, behavioral tests were performed after 8 weeks of daily SI of D-gal. All mice were fed a standard pellet diet, and water was freely available (Fig. S1B). The dose of urolithin A used in the present study was determined according to a preliminary experiment in our lab.

At the end of the 8 weeks, all mice were euthanized, and the blood samples collected from the tail vein of the mice were prepared for measurements of biochemical parameters. Then, the brain tissues were isolated on ice. One part of the brain was cut longitudinally, fixed with $4 \%$ polyformaldehyde, and H\&Estained for pathological studies, whereas the other parts of the brain tissues were homogenized for further experiments. The blood samples and brain tissues were rapidly frozen and stored at $-80{ }^{\circ} \mathrm{C}$ until biochemical determinations were performed. All experiments were performed in compliance with the ARRIVE (Animal Research: Reporting In Vivo Experiments) guidelines.

Additionally, 2-month $(n=24)$ and 12-month $(n=24)$ old mice were used to investigate the mechanism of urolithin A. After oral administration with urolithin A $(150 \mathrm{mg} / \mathrm{kg}, n=12)$ for 2 months, the animals were sacrificed and Western blotting analysis was performed to measure the effect of urolithin A on the activation of the miR-34a-mediated SIRT1/mTOR signaling pathway.

\section{Behavioral Tests}

\section{Open-Field Test}

To evaluate the motor activity and exploratory behavior of mice, an open-field test (OFT) was conducted using our previously described protocol with slight modifications [18]. In brief, mice were placed individually in a square arena, after which the total time spent in the central zone and total number of squares crossed in the OFT were recorded during 5-min trials. The apparatus was cleaned with $70 \%$ ethanol solution between each test to eliminate possible odors left by other mice.

\section{Morris Water Maze Test}

To evaluate the spatial learning and memory capacity of the Dgal-induced aging mice in this study, we performed a Morris water maze test (MWM) according to a protocol described previously with some modifications [19]. The experimental equipment consisted of a circular water tank $(120 \mathrm{~cm}$ in diameter, $50 \mathrm{~cm}$ in height) that contained water $\left(22 \pm 1^{\circ} \mathrm{C}\right)$ to a depth of $25 \mathrm{~cm}$, which was divided into 4 quadrants (northeast (NE), northwest (NW), southeast (SE), and southwest (SW)) and rendered opaque by adding milk powder. A small platform $(10 \mathrm{~cm}$ in diameter, $22 \mathrm{~cm}$ in height) was placed at the center of the third quadrant and submerged $1.5 \mathrm{~cm}$ below the surface of the water in a fixed location. The MWM procedure consisted of a place for the navigation training trial and a probe test. During the learning and memory training period, each mouse received 4 tests per day for 4 consecutive days. Once the mice were placed in the water maze, the animals were artificially guided to the hidden platform if they could not find it in $60 \mathrm{~s}$ and then stayed on the platform for $15 \mathrm{~s}$ to memorize its location. After the training trial, the probe test without the platform was conducted for $60 \mathrm{~s}$ in the tank.

\section{Object-Place Recognition}

To test a short-term object-place recognition memory, a modified version of the standard object recognition task was performed, according to previous studies [20]. Before the beginning of the experiment, each animal was allowed to adapt for $10 \mathrm{~min}$ in an open box $(70 \times 50 \times$ $40 \mathrm{~cm}$ ) and then started training at 30-min intervals during the course of testing. Object-place recognition training and testing took place in a quadratic dark gray box $(80 \times 80 \mathrm{~cm}$ $\mathrm{W}, 40 \mathrm{~cm} \mathrm{H}, \mathrm{PVC}$ ), and the time given to each individual rat was $10 \mathrm{~min}$. In the training session, 2 identical plastic objects (object A, $2.5 \times 2.5 \times 5 \mathrm{~cm}$ ) were placed in the apparatus diagonally at a distance of approximately $10 \mathrm{~cm}$ away from the wall. There was a 1-day rest interval between the training session and testing session. During the testing session, 2 other identical objects (objects A and B; cube $\mathrm{B}, 5 \times 5 \times 5 \mathrm{~cm}$ ) were placed in a symmetrical position approximately $10 \mathrm{~cm}$ from the wall and the animals. All of the objects are similar in texture, color, and size, but were distinctive in shape, and each type was filled with sand of a different color. The measures for trial and test 
were the time spent on each object by each animal. The objects and open field were cleaned thoroughly between trials with $60 \%$ ethanol solution.

\section{Body Weight Measurement and Tissue Sampling}

After a 24-h fasting period, body weight was monitored and recorded. After the behavioral tests, all mice were deeply anaesthetized and sacrificed by cervical dislocation. The brain tissues were promptly dissected and perfused with ice-cold $0.1 \mathrm{M}$ phosphate-buffered saline (PBS) solution. The organs were then washed with $0.9 \%$ normal saline $(w / v=1: 5)$ until no blood was found. Then, the brain tissues were weighed and homogenized in $50 \mathrm{mM}$ cold PBS ( $\mathrm{pH}$ 7.4) followed by centrifugation at $10,000 \mathrm{~g}$ for $10 \mathrm{~min}$ at $4{ }^{\circ} \mathrm{C}$. The collected supernatants were stored and used for biochemical evaluation. The tissue protein concentration was measured with the bicinchoninic acid (BCA) method.

To investigate the effect of urolithin $\mathrm{A}$ on the brain index in aging mice, the organ coefficient was calculated. Brain index (BI) was the ratio of brain weight and body weight, and the coefficient was calculated according to the following equation: coefficient $(\mathrm{mg} / \mathrm{g})=$ organ weight $(\mathrm{mg}) /$ body weight $(\mathrm{g})$.

\section{Immunohistochemistry}

Immunohistochemistry was used to detect the expression of glial fibrillary acidic protein (GFAP) in the brain tissue. Immunohistochemistry was performed as previously described [21]. Paraffin-embedded tissues were cut into 4- $\mu \mathrm{m}$ section, mounted on glass slides, and stained using indirect immunoperoxidase. The paraffin sections were baked in an oven at $65{ }^{\circ} \mathrm{C}$ for $24 \mathrm{~h}$, then rinsed with PBS 3 times, each time for $5 \mathrm{~min}$. The well-washed section was placed in the ethylene diamine tetraacetic acid (EDTA) buffer and then heated to boiling in a microwave oven. This step was repeated for 4 times, each time for $6 \mathrm{~min}$. After natural cooling, the sections were washed with PBS 3 times, each time for $3 \mathrm{~min}$. The sections were blocked with $3 \% \mathrm{H}_{2} \mathrm{O}_{2}$ for $30 \mathrm{~min}$ at room temperature, then washed with PBS 3 times and incubated with 5\% bovine serum albumin (BSA) for $20 \mathrm{~min}$. Next, the slices were incubated overnight at $4{ }^{\circ} \mathrm{C}$ with $50-\mu \mathrm{L}$ diluted anti-GFAP antibody, then incubated with 50 to $100-\mu \mathrm{L}$ goat anti-rabbit secondary antibody at $4{ }^{\circ} \mathrm{C}$ for $50 \mathrm{~min}$. The sections were washed with PBS 3 times and incubated in 3,3'-diaminobenzidine $(\mathrm{DAB})$ and then were washed with distilled water. The Image Pro-Plus version 6.0 software (IPP, version 6.0, Media Cybernetics, Silver Spring, MD, USA) was used for analysis of the positively stained area. The cell membrane or cytoplasm of neurons represented brown as strong positive staining, and the staining density revealed the GFAP protein expression level.

\section{Histomorphological Evaluations}

Fresh brain biopsy specimens were collected from mice and fixed in $4 \%$ polyformaldehyde $(\mathrm{pH} 7.4)$ at $4{ }^{\circ} \mathrm{C}$ for $4 \mathrm{~h}$, incubated overnight at $4{ }^{\circ} \mathrm{C}$ in $100 \mathrm{mM}$ sodium phosphate buffer (pH 7.4) containing 30\% sucrose, and embedded in paraffin. Then, sections (4- $\mu \mathrm{m}$-thick) were stained with haematoxylin and eosin (H\&E) and Nissl staining according to standard procedure and examined under a microscope.

\section{Biochemical Analysis}

The levels of T-SOD (U/mg protein), CAT (U/mg protein), GSH-Px (U/mg protein), T-AOC (U/mg protein), and MDA (nmol/mg protein) and the activity of AChE (U/mg protein) and MAO (U/mg protein) in the whole brain tissue were measured by spectrophotometry with commercially available kits (Nanjing Jiancheng) at the Department of Pharmacy, Renmin Hospital of Wuhan University. A Shimadzu 2450 UV spectrophotometer (UV2450; Shimadzu, Kyoto, Japan) was used for the analysis of these samples, and the reagent kits were SOD450 (SOD), CAT405 (CAT), GSH-Px412 (GSH-Px), TAOC520 (T-AOC), MDA532 (MDA), AChE412 (AChE), and MAO242 (MAO). The levels of the pro-inflammatory cytokines TNF- $\alpha(\mathrm{pg} / \mathrm{mL}), \mathrm{IL}-1 \beta(\mathrm{pg} / \mathrm{mL})$, and IL-6 (pg/ $\mathrm{mL}$ ) were determined in a hippocampal tissue supernatant using standard ELISA kits (Santa Cruz, CA), and the evaluation was performed with a Shimadzu (UV2450) spectrophotometer. All biochemical analyses followed the manufacturer's protocols.

\section{Real-Time Polymerase Chain Reaction (RT-PCR)}

The extraction of brain tissue samples from mice was similar to that described in the previous section. The frozen tissue samples were retrieved and then homogenized in 1-mL TRIzol reagent (Gibco-Invitrogen, Carlsbad, USA). Total mRNA was extracted using the RNeasy Lipid Tissue Mini Kit and reverse-transcribed into cDNA using oligo(dT) 18 primers and AMV reverse transcriptase (Gibco). Quantitative gene expression was determined by Power SYBR Green PCR Master Mix in an IQ 5.0 optical system. The fold change for each miRNA was calculated using the comparative $\mathrm{Ct}(\Delta \Delta \mathrm{Ct})$ equation after normalization to the reference expression of U6 small nuclear RNA. The relative miR-34a expression was calculated from 3 different experiments. The primer sequences used in this study are listed as follows: miR-34a forward: 5'-ACAC TCCAGCTGGGTGGCAGTGTCTTAGCT-3', reverse: 5'CTCAACTGGTGTCGTGGAGTCGGCAATTCAGT TGAGACAACCAG-3'. 


\section{Western Blot Analysis}

Brain tissue homogenates were lysed by RIPA (radioimmunoprecipitation assay) lysis buffer, which enables the extraction of cytoplasmic, membrane, and nuclear proteins. The tissue homogenates were collected by centrifugation at $12,000 \mathrm{rpm}$ for $30 \mathrm{~min}$ at $4{ }^{\circ} \mathrm{C}$, and the supernatant was saved and used for concentration determination with a bicinchoninic acid (BCA) protein assay kit. Equal amounts of protein $(100 \mu \mathrm{g})$ were isolated by $10 \%$ sodium dodecyl sulfate-polyacrylamide gel electrophoresis (SDS-PAGE) and then transferred to a polyvinylidene difluoride (PVDF) membrane, which was blocked with 5\% nonfat dry milk in Tris-buffered saline with Tween 20 (TBST, $150 \mathrm{mM} \mathrm{NaCl}, 20 \mathrm{mM}$ Tris- $\mathrm{HCl}$, and $0.1 \%$ Tween 20, $\mathrm{pH} 7.5$ ) at room temperature for $1 \mathrm{~h}$. Membranes were incubated overnight at $4{ }^{\circ} \mathrm{C}$ with the respective antibodies diluted at 1:1000 and then incubated with secondary antibody HRPlabeled goat anti-rabbit $\operatorname{IgG}(1: 2000)$ or anti-mouse $\operatorname{IgG}$ (1:4000) for $1.5 \mathrm{~h}$ after being washed 3 times with TBST. Finally, images of the target protein were developed, collected, and analyzed with the LAS4000 mini gel-imaging system (General Electric, Boston, MA).

\section{Statistical Analyses}

All data were expressed as the mean \pm S.D.. The statistical analyses were performed using the SPSS 13.0 statistical software package (SPSS Inc., Chicago, IL). One-way analysis of variance (ANOVA) was used to perform comparisons among the different groups, and $P<0.05$ was considered statistically significant.

\section{Results}

\section{Effect of In Vitro Treatment of Urolithin A on PC12 Cells}

\section{Urolithin A Protected PC12 Cells Against $\mathrm{H}_{2} \mathrm{O}_{2}$-Induced Cytotoxicity and Viability}

To assess the cytotoxic and neuroprotective effects of urolithin A on $\mathrm{PC} 12$ cells, cell viability and cytotoxicity assays were performed. As shown in Fig. S2A, compared with the viability of the control group, the viability of $\mathrm{PC} 12$ cells exposed to $\mathrm{H}_{2} \mathrm{O}_{2}$ was significantly $(P<0.01)$ decreased; however, cells treated with urolithin A significantly inhibited this decrease (all $P<0.01)$ with a significant dose-effect relationship. Furthermore, the results of the cytotoxicity assay (Fig. S2B) showed that treatment with $\mathrm{H}_{2} \mathrm{O}_{2}$ caused a significant increase in LDH release compared to the control group $(P<0.01)$, and urolithin A significantly attenuated this increase in LDH activity (all $P<0.01$ ). These data indicated that urolithin A can exert a protective effect on $\mathrm{H}_{2} \mathrm{O}_{2}$-induced $\mathrm{PC} 12$ cell oxidative injury.

\section{The Effects of Urolithin A on the Apoptosis of PC12 Cells Induced by $\mathrm{H}_{2} \mathrm{O}_{2}$}

To study and confirm whether urolithin A can inhibit the apoptosis of $\mathrm{PC} 12$ cells induced by $\mathrm{H}_{2} \mathrm{O}_{2}$, an Annexin VFITC apoptosis detection kit was used. The results of apoptosis experiments showed that exposure to $\mathrm{H}_{2} \mathrm{O}_{2}$ for $2 \mathrm{~h}$ resulted in evident apoptosis with an apoptosis rate of $73.45 \%$ compared to the control group $(P<0.01$, Fig. S3). However, pretreatment with urolithin A significantly decreased the apoptosis rate (all $P<0.01$ ), and the urolithin A group with a concentration of $50 \mu \mathrm{g} / \mathrm{mL}$ had the best effect on alleviating cell apoptosis induced by $\mathrm{H}_{2} \mathrm{O}_{2}$. These findings demonstrated that apoptosis in $\mathrm{PC} 12$ cells induced by $\mathrm{H}_{2} \mathrm{O}_{2}$ was effectively reversed by urolithin $\mathrm{A}$ treatment.

Furthermore, the effect of urolithin $\mathrm{A}$ on the protein expression of Bcl-2 and cleaved caspase-3 in $\mathrm{H}_{2} \mathrm{O}_{2}$-induced $\mathrm{PC} 12$ cell oxidative injury process was observed. As indicated in Fig. S4, treatment with $\mathrm{H}_{2} \mathrm{O}_{2}$ significantly increases the expression level of Bcl-2 and increases in caspase-3 activation when compared to the vehicle control (both $P<0.01$ ). However, the protein expressions of caspase- 3 and Bcl-2 were markedly regulated after $24 \mathrm{~h}$ of treatment with urolithin A (both $P<0.01$ ).

\section{Effect of In Vivo Treatment of Urolithin A on D-Gal-Induced Aging Mice}

\section{Effect of Urolithin A on Body Weight and Brain Index}

After 4 weeks of establishment of aging model, the mice in the normal group were active, were sensitive to sound and light, and possessed white and shiny hair and their feces were granular, whereas the mice in the model group were gaunt, listless, and unresponsive and their hair was lusterless. However, these deteriorated symptoms alternated in mice of urolithin A group. It was observed that there was a significant decrease in the body weight and brain index in D-gal-treated model group when compared with that in the control group when the experiment ended $(P<0.05$ or $P<0.01$, Fig. 1A, B). After 8 weeks of treatment with urolithin A, the brain index and weight of mice were significantly improved $(P<0.05$ or $P<0.01)$.

\section{Effect of Urolithin A on Behavioral Test of Mice}

\section{Open-Field Activity}

An open-field arena was used to evaluate the effect of urolithin $\mathrm{A}$ on the spontaneous locomotion of aging mice 
a

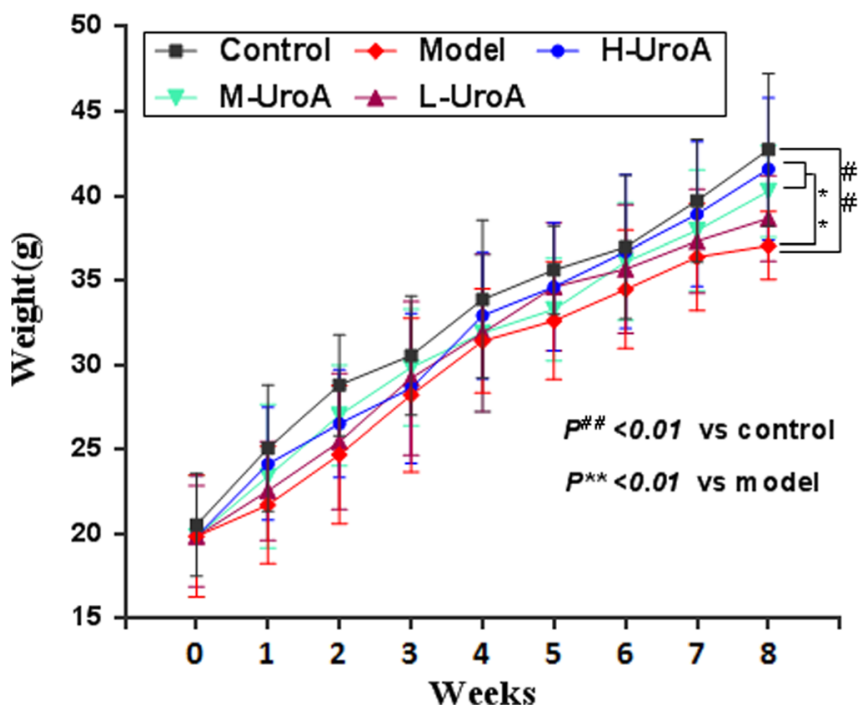

Fig. 1 The effect of urolithin A on body weight and brain index in aging mice. (A) Effect of urolithin A on body weight in D-gal-induced aging mice. (B) Effect of urolithin A on brain index in D-gal-induced aging

induced by D-gal. As shown in Fig. S5, there was a significant decrease in the number of squares crossed and the time spent in the central square with D-gal-treated mice versus the control animals $(P<0.01)$, which was considered a spontaneous activity defect. However, these spontaneous activity defects were significantly ameliorated by all 3 tested doses of urolithin A in our study (all $P<0.01$ ).

\section{Morris Water Maze}

To assess the effect of urolithin A on spatial learning and memory in the aging model mice, the Morris water maze was used in this study. As a result, chronic D-gal administration resulted in a significantly longer latency to reach the platform $(P<0.01)$, and treatment with urolithin A significantly decreased the escape latencies $(P<0.01)$ during the training period (Fig. S6A and S6B). In the spatial probe trial, D-gal-treated aging mice crossed into the former location of the platform fewer times $(P<0.01$, Fig. S6D) and spent a significantly decreased time in the goal area $(P<0.01$, Fig. S6C) compared with the control group. Interestingly, mice in the D-gal treatment plus urolithin A group displayed a remarkable reversion in these poor conditions. These findings demonstrated that urolithin A could restore the D-gal-induced deficit in memory and learning abilities. In addition, the aging mice in the model group displayed increased swimming speed compared with the other groups, but the difference was not significantly different (data not presented).

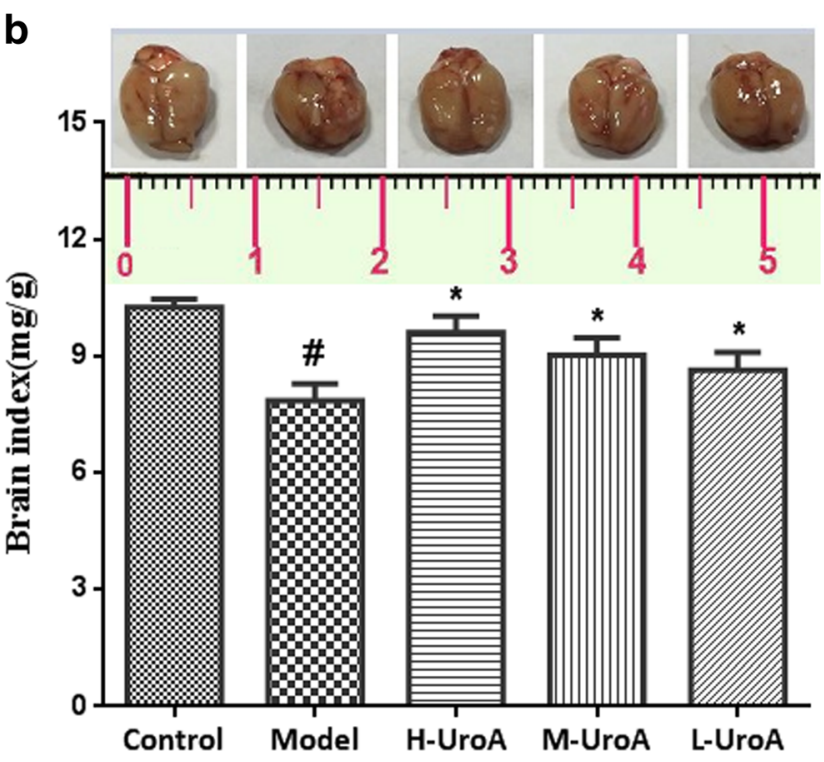

mice. All data are expressed as means \pm S.D. $(n=12) .{ }^{\#} P<0.05$ and ${ }^{\# \# P} P<0.01$ versus the control group; $* P<0.05$ and $* * P<0.01$ versus the model group

\section{Object-Place Recognition Test}

The results of the object-place recognition test for all groups are illustrated in Fig. S7. The model group displayed a significant deficit in object recognition learning caused by D-gal, as indicated by a reduced object exploration time (s) of spatially stationary and spatially displaced objects in the test trial compared with the control group $(P<0.01)$. In contrast, urolithin A administration significantly reversed these alterations in the object-place recognition task (all $P<0.01$ ), suggesting that urolithin A can significantly ameliorate D-gal-induced spatial learning and memory impairments.

\section{The Effect of Urolithin A on Biochemical Parameters in Mice}

\section{Effects of Urolithin A on AChE and MAO Activities in Whole Brain}

As shown in Fig. S8, the activity of AChE and MAO in the model group was significantly higher than that in the control group (both $P<0.01$ ). As a result, after supplementation with urolithin $\mathrm{A}$, the levels of $\mathrm{AChE}$ and MAO were significantly lowered in the whole brain of aging mice induced by D-gal when compared with the whole brain of the model group (all $P<0.01$ ). These findings showed that urolithin A could improve cognitive impairment. 
Effect of Urolithin A on SOD, CAT, GSH-Px, and T-AOC Activities and MDA Levels in the Brain

To investigate the effects of urolithin A on D-gal-induced oxidative stress, the activity of anti-oxidant enzymes and the content of anti-oxidants were measured. As shown in Fig. S9, there was a significant decrease in CAT, GSH-Px, SOD, and T-AOC activities, and a remarkable increase in MDA levels in brain tissues of D-gal-treated mice compared with the control animals (all $P<0.01$ ). However, the reductions in the activities of enzymatic or nonenzymatic enzymes in brain tissues could be significantly reversed by treating aging mice with urolithin A. These results suggested that urolithin A could attenuate D-gal-induced oxidative stress to protect brain tissues from damage.

\section{TNF- $\alpha$, IL-1 $\beta$, and IL-6 Determination}

As shown in Fig. S10, the expression of inflammatory mediators of TNF- $\alpha$, IL- 6 , and IL- $1 \beta$ in brain tissue increased significantly after modeling, which suggested that administration of D-gal could lead to chronic inflammatory conditions in aging mice. More importantly, the levels of the 3 proinflammatory cytokines were markedly decreased by treatment with different concentrations of urolithin A $(50 \mathrm{mg} / \mathrm{kg}$, $100 \mathrm{mg} / \mathrm{kg}$, or $150 \mathrm{mg} / \mathrm{kg}$ ) compared with those of the aging group activated by D-gal $(P<0.05$ or $P<0.01)$.

\section{Histological Findings}

Multiple lines of evidence have shown that D-gal injection can cause brain injury and dysfunction, as well as changes in brain morphology. H\&E and Nissl staining was used to analyze the histopathological features of mouse brain tissue in this study. As seen with H\&E staining (Fig. 2A), pyramidal cells, karyopyknosis, disorganized nerve fibers with irregular neurons, and an expended gap of neurons were observed in the CA3 region of D-gal-induced aging mice. However, urolithin A treatment reversed these pathological changes, and the high-dose urolithin A $(150 \mathrm{mg} / \mathrm{kg})$ treatment provided a maximum protective effect with minor changes observed in the morphological structure of the treatment group, which was almost identical to that of the normal control groups.

In the Nissl staining assay, it was observed that neurons were significantly damaged or lost in the CA3 region of the hippocampal tissue (Fig. 2B). The results revealed that the number of neurons in the hippocampus was significantly decreased in the model group; moreover, the population of Nissl bodies in aging model mice was also significantly decreased. However, the number of neurons in the hippocampus treated with urolithin A clearly recovered $(P<0.01$, Fig. $2 C)$. These results showed that urolithin A treatment produced a significant effect on ameliorating hippocampal injury caused by D-gal.

\section{Protective Effects of Urolithin A Against D-Gal-Induced Brain Aging via Regulating miR-34a-Mediated SIRT1/mTOR Signaling Pathway}

\author{
Urolithin A Downregulated D-Gal-Induced miR-34a \\ in the Hippocampal Tissue
}

miR-34a mRNA expression was examined in the hippocampal tissue before and after urolithin A intervention via quantitative real-time polymerase chain reaction. The present study showed that the expression of miR-34a in the hippocampal tissue varied in each group of mice. As described in Fig. S11, miR-34a expression was significantly upregulated in aging mice induced by D-gal compared with that in the control group $(P<0.01)$. Conversely, miR-34a mRNA expression was notably decreased after the administration of urolithin A for 8 weeks. These findings indicated that the upregulation of miR-34a was positively correlated with brain aging in D-galinduced mice.

\section{Urolithin A Ameliorated D-Gal-Induced Aging of Hippocampal Neurons Through Upregulating SIRT1 and Downregulating p53/p21}

In this study, we investigated whether $\mathrm{p} 53$ could induce miR34a expression and whether this increase was modulated by urolithin A, and the protein expression of p-p53, p53, and p21 was detected using Western blotting analysis. As indicated in Fig. 3A, a positive correlation was observed among p53, p21, and miR-34a; in contrast, these changes were significantly alleviated by urolithin A treatment in a concentrationdependent manner (all $P<0.01$ ).

To test whether the inhibition of miR-34a by urolithin A was specifically modulated by SIRT1 signaling, we also determined the effect of urolithin A on SIRT1 protein expression by Western blot analysis. Western blot analysis showed that SIRT1 protein expression was markedly decreased in mice with aging induced by chronic treatment with D-gal, whereas urolithin A significantly reversed the decreased expression of SIRT1 (all $P<0.01$ ). These data suggest that urolithin A is associated with the regulation of SIRT1 and p53 phosphorylation in aging mice.

\section{Urolithin A Suppressed D-Gal-Induced Apoptosis and Rescued Dysfunctional Autophagy}

As shown in Fig. 3B, D-gal caused an obvious increase in cleaved caspase- 3 and a significant decrease in Bcl-2 in the hippocampus of D-gal-administered mice compared with those of the normal group $(P<0.01)$. In contrast, cleaved caspase- 3 expression significantly decreased and Bcl-2 expression was notably upregulated in the urolithin A treatment group compared with those in the model group (all $P<0.01$ ). 

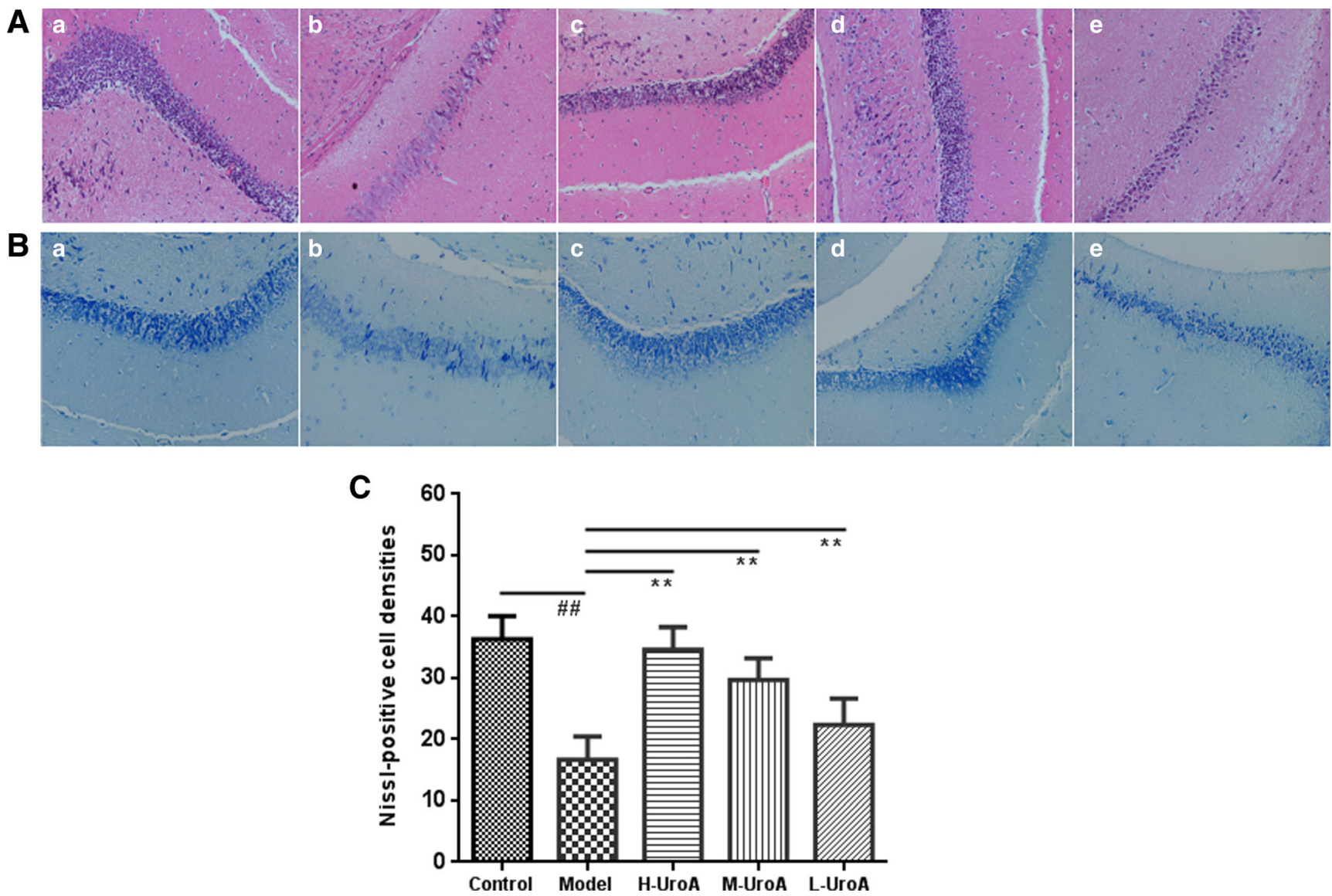

Fig. 2 Effect of urolithin A treatment on brain histopathological alterations with H\&E staining (A) and Nissl staining (B) (magnification $\times 200$ ): (a) control group; (b) model group; (c) H-UroA group; (d) MUroA group; (e) L-UroA group. The population of Nissl-positive cells in

hippocampus tissues with Nissl staining $(\mathbf{C})$. Data are presented as mean \pm S.D. from each group $(n=12)$. ${ }^{\#} P<0.05$ and ${ }^{\# \#} P<0.01$ versus the control group; $* P<0.05$ and $* * P<0.01$ versus the model group

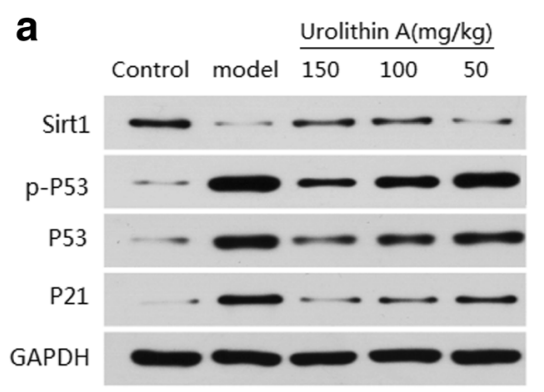

b

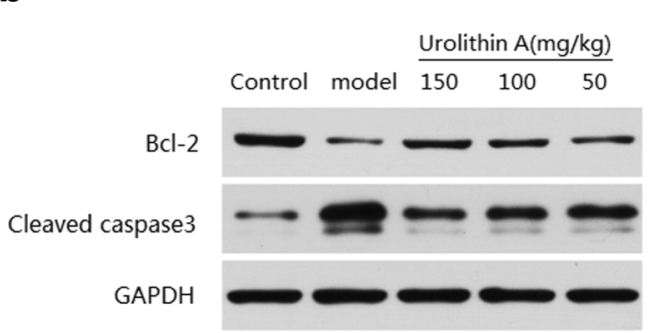

Fig. 3 The effect of urolithin A on SIRT1/p53 signaling in the hippocampus tissue of D-gal-induced aging mice (A). Urolithin A attenuated the excessive apoptosis in the hippocampus tissue of D-gal-induced aging
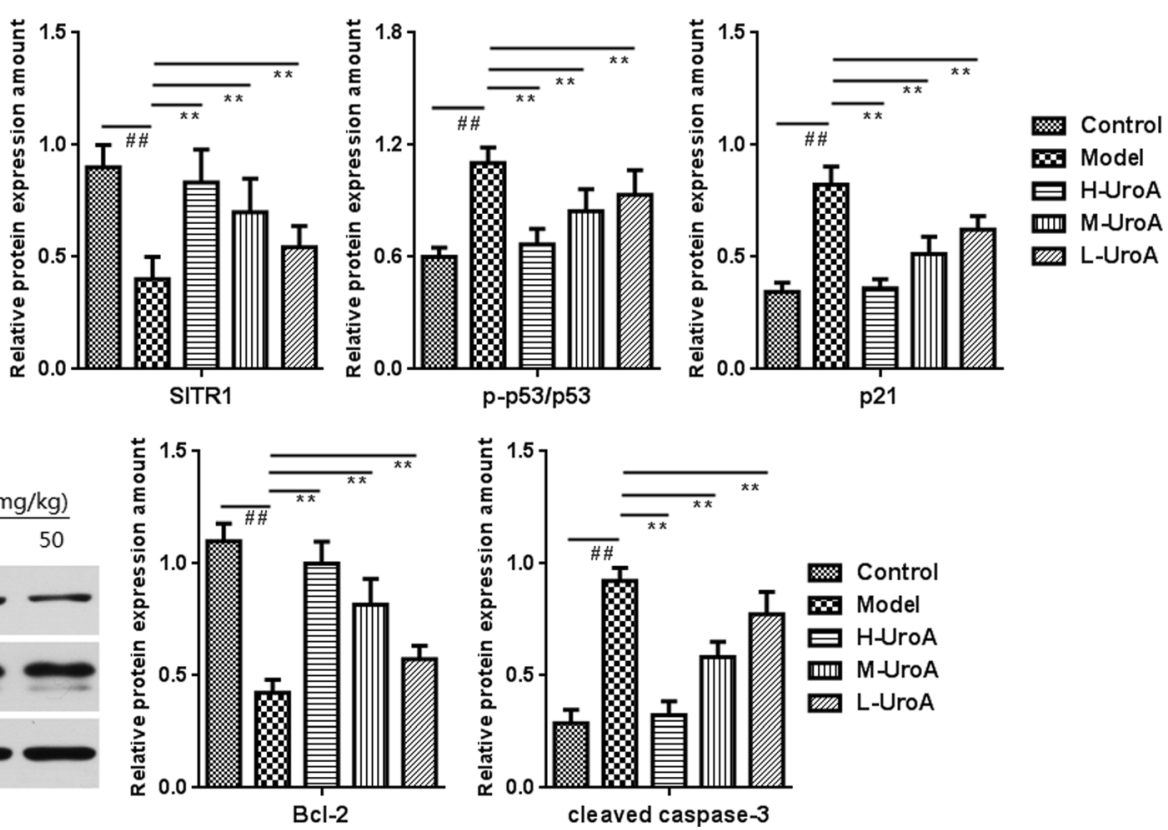

mice (B). Data are presented as mean \pm S.D. from each group $(n=12)$, ${ }^{\#} P<0.05$ and ${ }^{\# \#} P<0.01$ versus the control group; ${ }^{*} P<0.05$ and $* * P<0.01$ versus the model group 
These data demonstrate that urolithin A can exert an antiapoptotic function to protect aging mice against D-gal-induced cell damage.

Moreover, the expression levels of the proteins associated with the functional status of autophagy were detected. As shown in Fig. 4A, compared with those in the control group, the expression of Atg7 protein and the LC3-II/LC3-I ratio in the D-gal-induced aging model group were dramatically decreased $(P<0.01)$, suggesting that dysfunctional autophagy was evidently induced in the hippocampal tissue due to $\mathrm{D}$ gal administration. However, the downregulation of autophagy-related proteins was significantly rescued by pretreatment with urolithin A (all $P<0.01$ ). Furthermore, it was observed that the p62 level in the hippocampal tissues of aging mice induced by D-gal was significantly increased, and these increases were attenuated by treatment with urolithin A.

\section{Urolithin A Attenuated D-Gal-Induced Astrocyte Activation in the Hippocampal Tissue}

As illustrated in Fig. 4B, the GFAP expression in mice of the D-gal-induced aging model group was significantly increased compared with that of the control group $(P<0.01)$. However, after treatment with urolithin A, GFAP was significantly downregulated (all $P<0.01$ ). Furthermore, the immunohistochemical results indicated that GFAP levels are significantly higher in the D-gal model group than in the control group (Fig. S12A). Interestingly, the administration of urolithin A resulted in a significant decrease in the number of GFAP
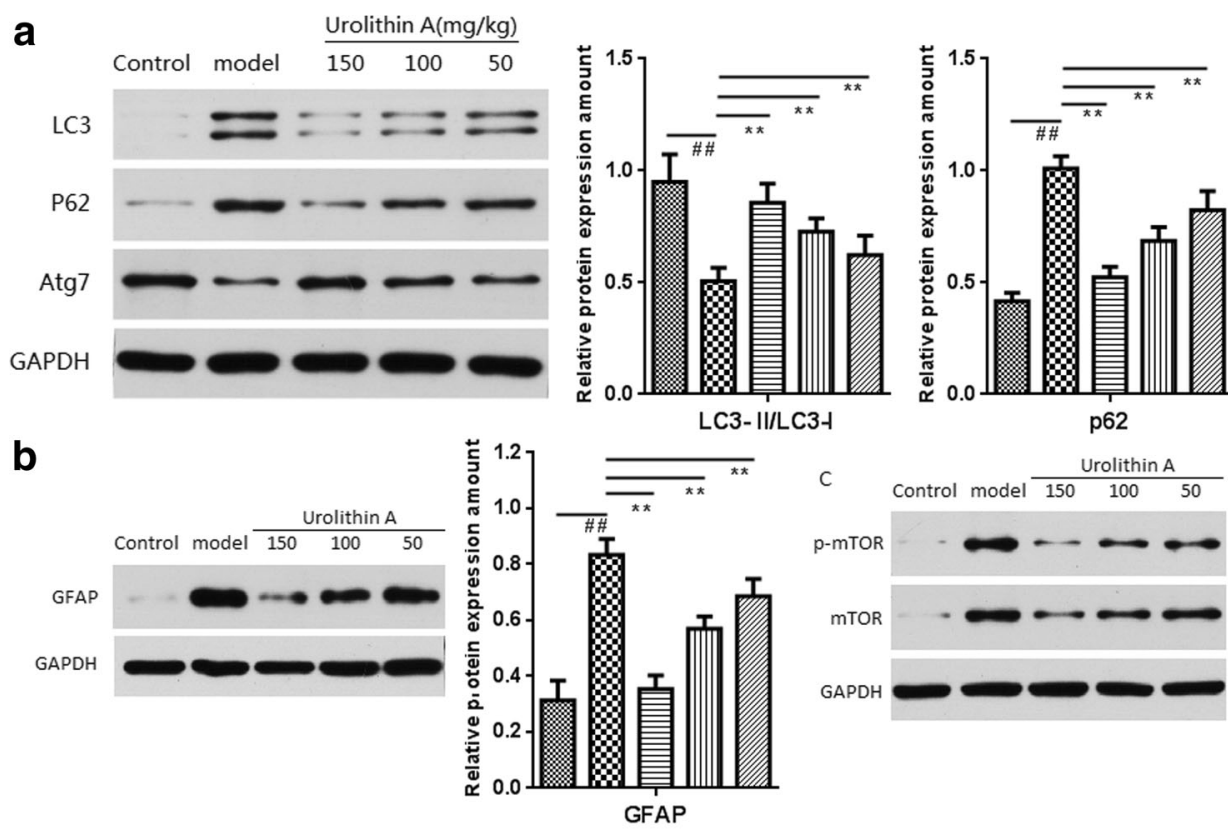

Fig. 4 Urolithin A rescued the deficient autophagy in the hippocampus tissue of D-gal-induced aging mice (A). Urolithin A reduced the overexpression of GFAP in the hippocampus tissue of D-gal-induced aging mice (B). Urolithin A inhibited mTOR activation in the

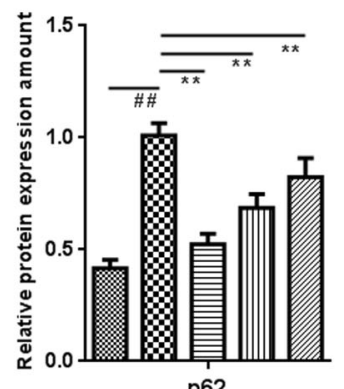
the model group immunoreactive astrocytes when compared with that of the model group $(P<0.01$, Fig. S12B). These findings reinforce the benefits of urolithin A on aging mice induced by D-gal to further support that urolithin A attenuates D-gal-induced astrocyte activation in the hippocampal tissue.

\section{Urolithin A Inhibited the mTOR Signaling Pathway in the Hippocampal Tissue of D-Gal-Induced Aging Mice}

To further assess whether modulation of mTOR occurs via urolithin A-mediated protective effects, we assessed the expression of mTOR by Western blot analysis. As shown in Fig. 4C, D-gal administration resulted in increased levels of mTOR phosphorylation at $\operatorname{Ser}^{2448}$ (p-mTOR) in the hippocampal tissue of D-gal-induced aging mice. In addition, urolithin A supplementation significantly reversed the increased mTOR expression level, indicating that the activation of autophagy occurred through inhibiting the effect of urolithin A on mTOR signaling.

\section{Effect of Urolithin A on Normal Young Mice and Natural Aging Mice}

To further study the effects of urolithin A on normal young mice and natural aging mice, 2-month-old and 12-month-old mice were treated with urolithin A for 2 months, and the expression of miR-34a in the hippocampal tissue was measured in these mice. As shown in Fig. S13, urolithin A significantly decreased the miR-34a expression levels in both the 2-
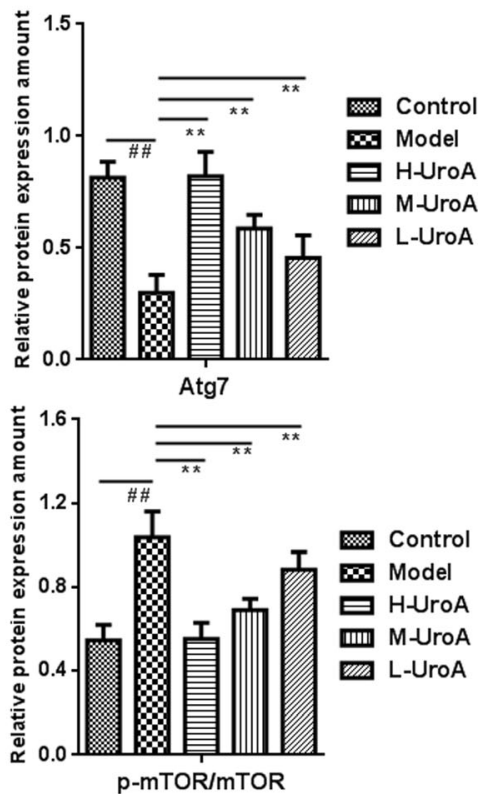

hippocampus tissue of D-gal-induced aging mice $(\mathbf{C})$. Data are presented as mean \pm S.D. from each group $(n=12) .{ }^{\#} P<0.05$ and ${ }^{\# \#} P<0.01$ versus the control group; $* P<0.05$ and $* * P<0.01$ versus 
month-old and 12-month-old mice $(P<0.01$ and $P<0.01)$. Furthermore, we found that the 12-month-old mice experienced a significant increase in miR-34a expression compared with the 2 -month-old mice $(P<0.01)$. In addition, we also investigated the effect of urolithin $\mathrm{A}$ on the activation of miR-34a-mediated SIRT1/mTOR signaling pathway on normal young and natural aging mice. Similar to the results of Dgal-induced aging model experiment, urolithin A can upregulate SIRT1 and downregulate p53/p21 (Fig. 5A), suppress apoptosis (Fig. 5B) and rescue dysfunctional autophagy (Fig. 6A), attenuate astrocyte activation (Fig. 6B, Fig. S14), and inhibit the mTOR signaling pathway (Fig. 6C) in the hippocampal tissue of normal aging mice. These data confirmed the hypothesis that miR-34a expression levels in young rodents were lower than in their partners and urolithin A has a protective effect on brain aging mice via the activation of the miR-34a-mediated SIRT1/mTOR signaling pathway.

\section{Discussion}

Natural aging is a progressive process related to a series of morphologic and behavioral changes including observed neurodegenerative dysfunction, such as in motor and cognitive performance [22]. Drug discovery from natural bioactive substances against neurodegeneration may be an effective intervention to delay brain aging. In the present study, we first evaluated the neuroprotective effects of urolithin A on $\mathrm{H}_{2} \mathrm{O}_{2}$-induced oxidative injury and apoptosis in $\mathrm{PC} 12$ cells in vitro. We then established an animal aging model by chronic injection of D-gal $\left(150 \mathrm{mg} \mathrm{kg} \mathrm{day}{ }^{-1}\right)$ for 8 weeks in mice to assess the effects of urolithin A on spatial learning and memory function by using the Morris water maze, the openfield test, and the object-place recognition. Furthermore, we also investigated the action of urolithin A supplementation on excessive apoptosis and dysfunctional autophagy of hippocampal neurons and astrocyte activation associated with Dgal-induced brain aging. To our knowledge, this study provides evidence for the first time that 1) pretreatment with urolithin A attenuates $\mathrm{H}_{2} \mathrm{O}_{2}$-induced apoptosis in $\mathrm{PC} 12$ cells; 2) urolithin A treatment ameliorates the cognitive deficits induced by D-gal in mice; and 3) urolithin A has a protective effect on neurological deficits in D-gal-induced aging mice. Moreover, urolithin A administration suppressed cell apoptosis and astrocyte activation and activated autophagy in the hippocampal tissue of aging mice.

Accumulating evidence has indicated that a number of miRNAs play a crucial role in modulating cellular senescence [23]. Previous studies have shown that miR-34a is upregulated in a variety of age-related diseases, including AD [24]. Based on the above research findings, we examined the activation of miR-34a mRNA expression in the brain during aging. Interestingly, our results showed that miR-34a levels increased in the D-gal-induced brain aging model, which is consistent with previous studies $[5,10]$, suggesting that elevated miR-34a is involved in common pathologic events of brain aging. Strikingly, urolithin A treatment significantly reversed the D-gal-induced upregulation of miR-34a, which prompted us to further explore the target gene of miR-34a in the rescue process of urolithin A treatment for brain aging. It has been
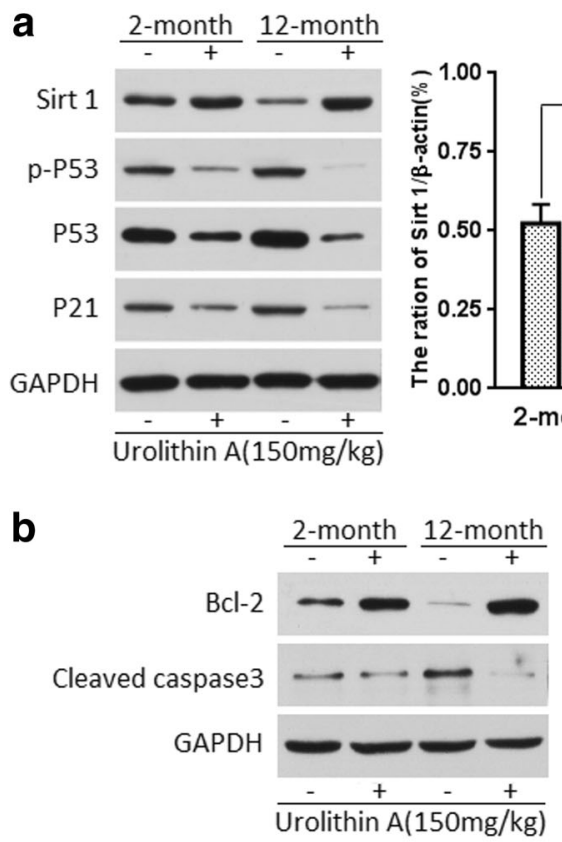
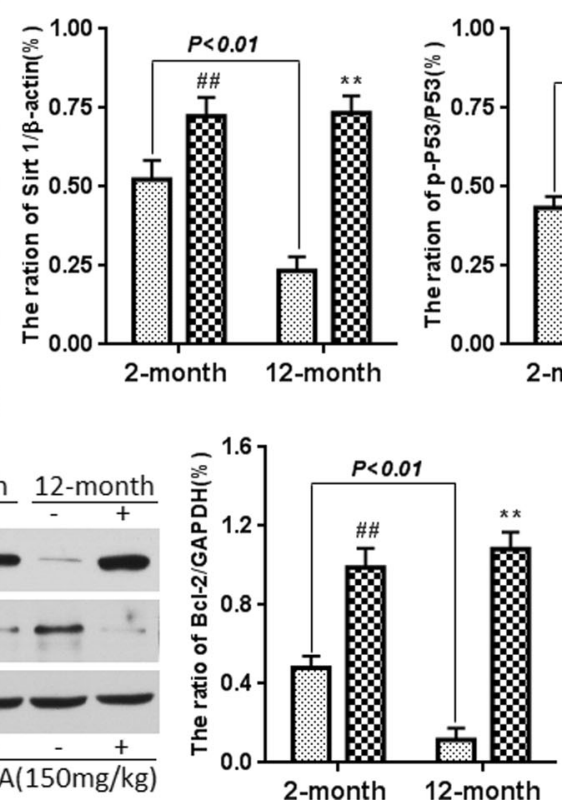
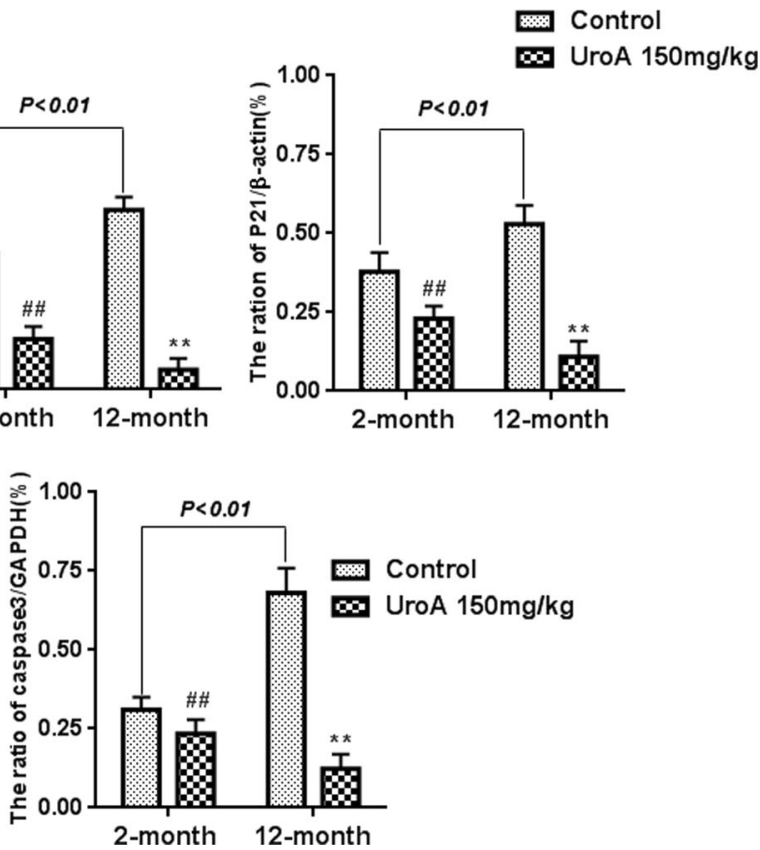

Fig. 5 The effect of urolithin A on SIRT1/p53 signaling in the hippocampus tissue of normal young mice and natural aging mice (A). Urolithin A attenuated the excessive apoptosis in the hippocampus tissue of normal young mice and natural aging mice $(\mathbf{B})$. Data are presented as mean \pm S.D. from each group $(n=12)$. ${ }^{\#} P<0.05$ and ${ }^{\# \#} P<0.01$ versus the control group; $* P<0.05$ and $* * P<0.01$ versus the model group 


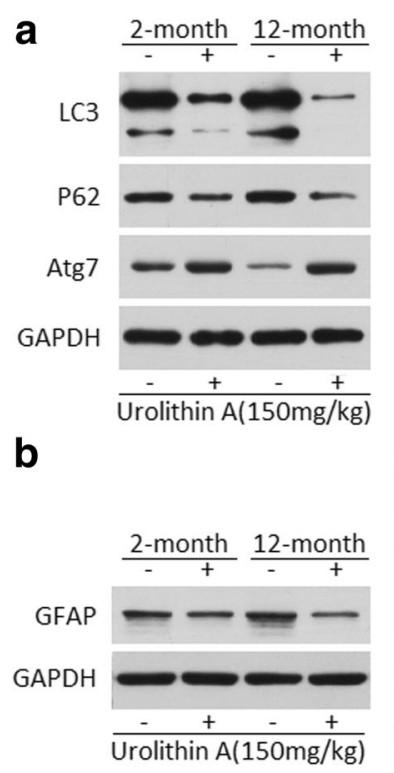

Fig. 6 Urolithin A rescued the deficient autophagy in the hippocampus tissue of normal young mice and natural aging mice (A). Urolithin A reduced the overexpression of GFAP in the hippocampus tissue of normal young mice and natural aging mice $(\mathbf{B})$. Urolithin A inhibited
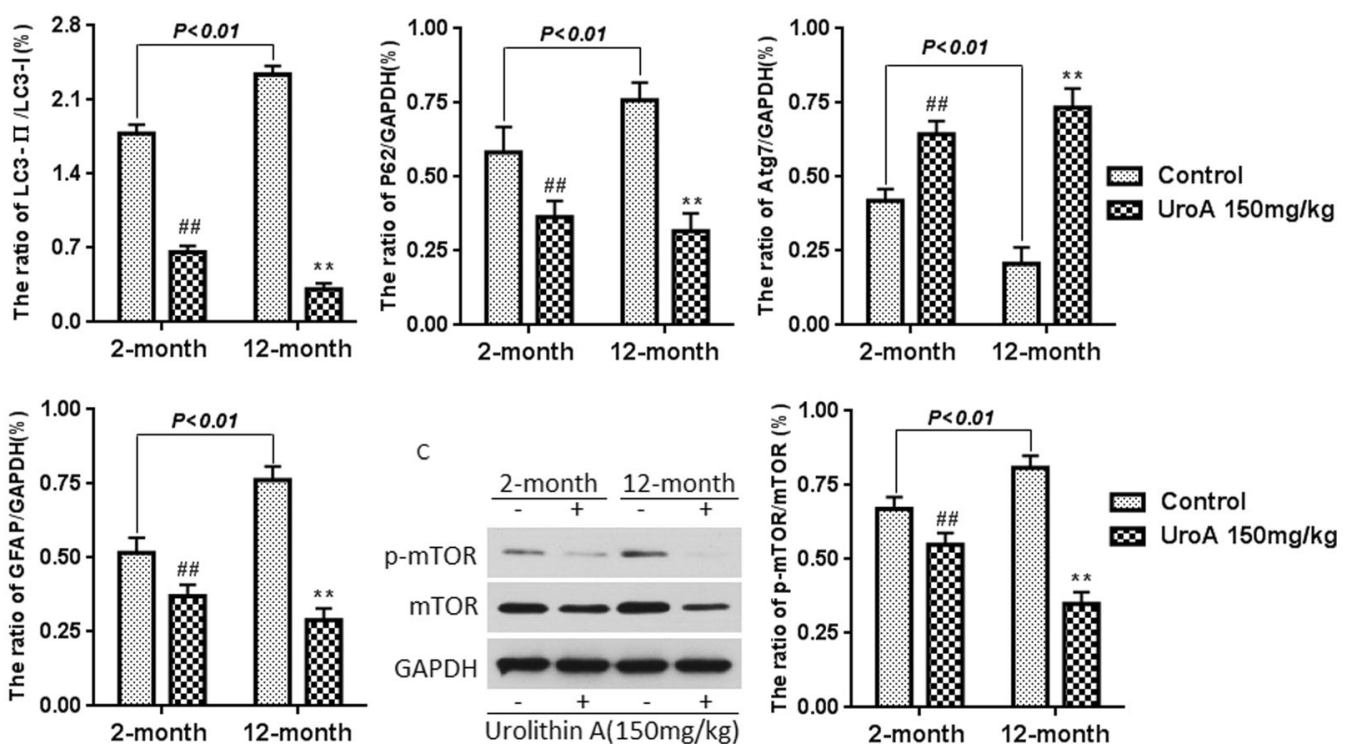

mTOR activation in the hippocampus tissue of normal young mice and natural aging mice $(\mathbf{C})$. Data are presented as mean \pm S.D. from each group $(n=12) .{ }^{\#} P<0.05$ and ${ }^{\# \#} P<0.01$ versus the control group; $* P<0.05$ and $* * P<0.01$ versus the model group

confirmed that $\mathrm{p} 53$ and $\mathrm{p} 21$ are related to the regulation of cell cycle, DNA repair, apoptosis, and other important biological functions, whereas SIRT6 acts as a negative regulator of cell senescence and can reduce the risk of aging-related diseases, especially disorders associated with brain dysfunction [25, $26]$. In the present study, we observed that D-gal administration in mice resulted in the overexpression of senescenceassociated signals including $\mathrm{p}-\mathrm{p} 53, \mathrm{p} 53$, and $\mathrm{p} 21$ and the downregulation of SIRT1 in the hippocampus, and urolithin A treatment reversed these changes, accompanied by a significant decrease in miR-34a expression. Therefore, the reduction of related proteins and genes associated with aging and/or longevity in the model could be compensated through the upregulation of SIRT1 induced by urolithin A intervention [27]. However, the mechanisms of protective effects of urolithin A against brain aging are not fully understood, and its effect on SIRT1 expression remains to be further explored.

It has been reported that oxidative stress is one of the key factors responsible for neuronal apoptosis, which is associated with natural aging $[15,17]$. As a result, cell apoptosis is markly increased in the hippocampal tissue of aging mice; in contrast, urolithin A can attenuate the D-gal-induced apoptosis. The results suggested that urolithin A may possess protective effects against D-gal-induced brain dysfunction. Thus, miR-34a may have been implicated not only as an initiator of the apoptotic signaling pathway but also as a promising therapeutic target in protecting neurons against death during the process of aging $[23,24]$. Moreover, the anti-apoptosis factor Bcl-2, which acted as an important target of miR-34a, has been evaluated, and D-gal led to a significant decrease in
Bcl-2 levels in aging mice. The reduction in Bcl-2 levels had been obviously upregulated by treatment with miR-34a, suggesting that miR-34a expression is strongly correlated with aging and that urolithin A can exert an anti-aging effect by inhibiting the expression of the apoptotic signaling pathway through miR-34a downregulation [28].

Several recent studies have found that activation of astrocytes appears to correlate with the accumulation of amyloid beta $(\mathrm{A} \beta)$ occurring early in the brain of human $\mathrm{AD}$ and in a mouse model of $\mathrm{AD}$, and both are accompanied by an increase in GFAP [21]. The findings reported here are consistent with previous characterizations that learning and memory deficits induced by long-term injection of D-gal (prominent changes of $\mathrm{AD}$ in the early stage) are relevant to increased GFAP levels as a marker of astrocyte activation in the AD mouse hippocampus, suggesting that astrocytes contribute to age-related cognitive dysfunction, including impairment of the hippocampus [29]. It is interesting to note that urolithin A can accomplish its neuroprotective function due to the effective inhibition of GFAP in the hippocampus of mice induced by D-gal upon urolithin A therapy.

It has been reported that miRNAs, through mRNA silencing, can affect the functioning of multiple downstream cell signaling pathways and can provide new avenues of research to complete recovery or dramatic improvement of human cognitive functions associated with many age-related diseases [6-8]. Autophagy, as a catabolic process that recycles macromolecules and organelles, is primarily responsible for sequestering cytoplasmic components for degradation/recycling and generating substrates to maintain cellular homeostasis and 
healthy neurons [30]. However, if autophagy is impaired or excessive, it can lead to the accumulation of ubiquitinated proteins and damaged organelles (such as uncoupled mitochondria) and even promote cell death by apoptosis or necrosis [31]. A decline in autophagic responses of cells and tissues has become the characteristic of aging in any complex system. A growing number of studies have demonstrated that dysfunction in autophagy has been implicated in the pathogenesis of neurodegenerative diseases, including AD and PD; thus, elevating miR-34a-mediated functional status of autophagy can be used for the therapeutic purposes in aging and chronic agerelated diseases $[6,32]$.

To further elucidate the functional status of autophagy in the hippocampus, the autophagic markers LC3II and p62, which are widely used for estimating autophagic activity, were analyzed by Western blotting in the present study. Western blot analysis revealed that D-gal led to a significant increase in p62 levels and downregulated LC3 and Atg7 in hippocampal neurons in the model group. The present findings confirmed that autophagy is inhibited in the hippocampus of Dgal-induced aging mice, which suggested that autophagy may be the partial mechanism underlying brain aging and the decline of age-related learning and memory [33].

It is now generally believed that autophagy and mTORmediated autophagy signaling pathways are considered to be major contributors to aging and many aging-associated pathologies [34]. It has been reported that upregulation of autophagy is a mechanism of delaying senescence and can also be inhibited through activation of the mTOR complex [35]. As expected, supplementation with urolithin A can deactivate the mTOR signaling pathway in a D-gal-induced mimetic mouse model with brain aging. Except for maintaining a functional energy charge, which is critical for metabolic homeostasis, the interactions of SIRT1 with FoxO and p53 signaling can also regulate both autophagic degradation and lifespan extension [36]. As explained in previous reports, some polyphenolic compounds and natural anti-oxidants such as resveratrol and grapes can significantly improve SIRT1 activities in vitro, thereby enhancing the activities and expression of SIRT2 to mimic calorie restriction and extend the lifespan of yeast by approximately $70 \%$ [37]. Thus, it suggested that small molecule activators of SIRT1, which function as a key target in neurological diseases, might be a promising therapeutic strategy to ameliorate the development of brain aging.

Furthermore, it has been demonstrated that SIRT1 can form a molecular complex with several essential components of autophagy machinery, including autophagy genes Atg5 and Atg7 [38]. Because SIRT1 can regulate multiple genes in autophagic signaling pathways and be activated when all activation conditions are met, SIRT1 regulation has been shown to play a significant role in the aging process [39]. These observations are generally consistent with our present data that urolithin A can clearly enhance the SIRT1 expression level and thereby contribute to the increased activity of $\operatorname{Atg} 7$ [40]. More importantly, there are multiple cellular targets for urolithin A, similar to other natural compounds. Our extensive studies have implied that $\mathrm{p} 53$ may act as a master repressor of autophagy in the regulation of autophagy [41]. Our previous studies revealed that the SIRT1-mediated signaling pathway interacts with p53 and experiences autophagic regulation, indicating that SIRT1 may also be able to regulate autophagic degradation via the $\mathrm{p} 53$ signaling pathway $[36,42]$. Consistent with the above literature reports, it was observed that urolithin A significantly decreased p-p53 and p53 expression, thus causing a reversal of brain aging induced by D-gal by enhancing autophagy.

\section{Conclusion}

In conclusion, the urolithin A-activated miR-34a-mediated SIRT1/mTOR signaling pathway (Fig. S15) plays an evident protective role in D-gal-induced aging-associated cognitive impairment. The underlying mechanism may be associated with the fact that urolithin A contributes to the induction of autophagy in the hippocampal tissues by restraining agingrelated astrocyte activation and inhibiting the activation of mTOR, as well as downregulating miR-34a, which provides a theoretical basis and support for the use of urolithin $\mathrm{A}$ as a promising therapeutic drug against brain aging and its associated neurological diseases.

Author Contributions Chen P and Dr. Zhou conceptualized and designed the study. Chen $\mathrm{P}$ performed the experiment, drafted the initial manuscript, and approved the final manuscript as submitted. FC Chen, JX Lei, and QL Li coordinated the project. All authors approved the final version of manuscript.

Funding Source This work was supported by grants from the National Natural Science Foundation of China (31770381) and Foundation of Health Commission of Hubei Province (2013Z-B04).

\section{Compliance with Ethical Standards}

All procedures were approved by the internal ethical committee of Wuhan University (IACUCWU protocol number: SCXK (E) 2013-0004).

Conflict of Interest The authors declare that they have no conflicts of interest.

\section{References}

1. Graham W V, Bonito-Oliva A, Sakmar T P. Update on Alzheimer's Disease Therapy and Prevention Strategies. Annu Rev Med 2017; 68: 413-430.

2. Wei J, Zhang G, Zhang X, Xu D, Gao J, Fan J, et al. Anthocyanins from Black Chokeberry (Aroniamelanocarpa Elliot) Delayed 
Aging-Related Degenerative Changes of Brain. J Agric Food Chem 2017; 65: 5973-5984.

3. Rathore S, Habes M, Iftikhar M A, Shacklett A, Davatzikos C. A review on neuroimaging-based classification studies and associated feature extraction methods for Alzheimer's disease and its prodromal stages. Neuroimage 2017; 155: 530-548.

4. Hussain R Z, Miller-Little W A, Lambracht-Washington D, Jaramillo TC, Takahashi M, Zhang S, et al. Laquinimod has no effects on brain volume or cellular CNS composition in the F1 $3 \times \mathrm{Tg}-\mathrm{AD} / \mathrm{C} 3 \mathrm{H}$ mouse model of Alzheimer's disease. J Neuroimmunol 2017; 309: 100-110.

5. Kou X, Liu X, Chen X, Li J, Yang X, Fan J, et al. Ampelopsin attenuates brain aging of D-gal-induced rats through miR-34amediated SIRT1/mTOR signal pathway. Oncotarget 2016; 7 : 74484-74495.

6. Lorenzen J M, Haller H, Thum T. MicroRNAs as mediators and therapeutic targets in chronic kidney disease. Nat Rev Nephrol 2017; 7: 286-294.

7. Long J M, Ray B, Lahiri D K. MicroRNA-153 Physiologically Inhibits Expression of Amyloid- $\beta$ Precursor Protein in Cultured Human Fetal Brain Cells and Is Dysregulated in a Subset of Alzheimer Disease Patients. J Biol Chem 2012; 287: 31298-31310

8. Long J M, Ray B, Lahiri D K. MicroRNA-339-5p down-regulates protein expression of $\beta$-site amyloid precursor protein-cleaving enzyme 1 (BACE1) in human primary brain cultures and is reduced in brain tissue specimens of Alzheimer disease subjects. J Biol Chem 2014; 289: 5184-5198.

9. Long J M, Maloney B, Rogers J T, Lahiri DK. Novel upregulation of amyloid- $\beta$ precursor protein (APP) by microRNA-346 via targeting of APP mRNA 5'-untranslated region: Implications in Alzheimer's disease. Mol Psychiatry 2019; 24: 345-363.

10. Kou X, Li J, Liu X, Chang J, Zhao Q, Jia S, et al. Swimming attenuates $\mathrm{D}$-galactose-induced brain aging via suppressing miR34a-mediated autophagy impairment and abnormal mitochondrial dynamics. J Appl Physiol 2017; 122: 1462-1469.

11. Singh R, Chandrashekharappa S, Bodduluri SR, Baby BV, Hegde B, Kotla NG, et al. Enhancement of the gut barrier integrity by a microbial metabolite through the Nrf2 pathway. Nat Commun 2019; 10: 89-106.

12. Cerdá B, Periago P, Espín JC, Tomás-Barberán FA. Identification of Urolithin A as a Metabolite Produced by Human Colon Microflora from Ellagic Acid and Related Compounds. J Agric Food Chem 2005; 53: 5571-5576.

13. Ryu D, Mouchiroud L, Andreux PA, Katsyuba E, Moullan N, Nicolet-Dit-Félix AA, et al. Urolithin A induces mitophagy and prolongs lifespan in $\mathrm{C}$. elegans and increases muscle function in rodents. Nat Med 2016; 2: 879-888.

14. Izzotti A, Cartiglia C, Steele V E, Silvio D F. MicroRNAs as targets for dietary and pharmacological inhibitors of mutagenesis and carcinogenesis. Mutat Res 2012; 751: 287-303.

15. Sang Y, Zhang F, Wang H, Yao J, Chen R, Zhou Z, et al. Apigenin exhibits protective effects in a mouse model of D-galactose-induced aging via activating the Nrf2 pathway. Food Funct 2017; 8: 23312340 .

16. Li WJ, Nie SP, Xie MY, Yu Q, Chen Y, He M. Ganoderma atrum polysaccharide attenuates oxidative stress induced by D-galactose in mouse brain. Life Sci 2011; 88: 713-718.

17. Chen P, Chen F, Zhou B. Antioxidative, anti-inflammatory and antiapoptotic effects of ellagic acid in liver and brain of rats treated by D-galactose. Sci Rep 2018; 8: 1465-1474.

18. Bodden C, Siestrup S, Palme R, Kaiser S, Sachser N, Richter SH Evidence- based severity assessment: Impact of repeated versus single open-field testing on welfare in C57BL/6J mice. Behav Brain Res 2017; 336: 261-268.
19. Jiang B, Song L, Huang C, Zhang W. P7C3 Attenuates the Scopolamine- Induced Memory Impairments in C57BL/6J Mice. Neurochem Res 2016; 41: 1010-1019.

20. Hall JH, Wiseman FK, Fisher EM, Tybulewicz VL, Harwood JL, Good MA. Tc1 mouse model of trisomy-21 dissociates properties of short- and long-term recognition memory. Neurobiol Learn Mem 2016; 130: 118-128.

21. Hong Z, Zhuo Q, Zhang J, Jiang B, Gao C, Wen Y. Shunaoxin dropping pill, a Chinese herb compound preparation, attenuates memory impairment in D-galactose-induced aging mice. Rsc Advances 2018; 8: 10163-10171.

22. AJ Ding, SQ Zheng, XB Huang, TK Xing, GS Wu, HY Sun, et al. Current Perspective in the Discovery of Anti-aging Agents from Natural Products. Nat Prod Bioprospect 2017; 7: 335-404.

23. Prathipati P, Nandi S S, Mishra P K. Stem Cell-Derived Exosomes, Autophagy, Extracellular Matrix Turnover, and miRNAs in Cardiac Regeneration during Stem Cell Therapy. Stem Cell Rev 2017; 13: 79-91.

24. Cui H, Ge J, Xie N, Banerjee S, Zhou Y, Liu RM, et al. miR-34a promotes fibrosis in aged lungs by inducing alveolar epithelial dysfunctions. Am J Physiol Lung Cell Mol Physiol 2017; 312: 415-424.

25. Farr JN , Xu M, Weivoda MM , Monroe DG, Fraser DG, Onken JL, et al. Targeting cellular senescence prevents age-related bone loss in mice. Nat Med 2017; 23:1072-1079.

26. Tasselli L, Zheng W, Chua K F. SIRT6: Novel Mechanisms and Links to Aging and Disease. Trends Endocrinol Metab 2017; 28: $168-185$.

27. Gao J, Zhou R, You X, Luo F, He H, Chang X, et al. Salidroside suppresses inflammation in a D-galactose-induced rat model of Alzheimer's disease via SIRT1/NF-?oB pathway. Metab Brain Dis 2016; 31: 771-778.

28. Ye Z, Fang J, Dai S, Wang Y, Fu Z, Feng W, et al. MicroRNA-34a induces a senescence-like change via the down-regulation of SIRT1 and up-regulation of p53 protein in human esophageal squamous cancer cells with a wild-type p53 gene background. Cancer Lett 2016; 370: 216-221.

29. Harach T, Jammes F, Muller C, Duthilleul N, Cheatham V, Zufferey V, et al. Administrations of human adult ischemia-tolerant mesenchymal stem cells and factors reduce amyloid beta pathology in a mouse model of Alzheimer's disease. Neurobiol Aging 2017; 51: 83-96.

30. Ho TT, Warr MR, Adelman ER, Lansinger OM, Flach J, Verovskaya EV, et al. Autophagy maintains the metabolism and function of young and old stem cells. Nature 2017; 543: 205-210.

31. Galluzzi L, Baehrecke EH, Ballabio A, Boya P, Bravo-San Pedro $\mathrm{JM}$, Cecconi F, et al. Molecular definitions of autophagy and related processes. Embo J 2017; 36: 1811-1836.

32. Menzies FM, Fleming A, Caricasole A, Bento CF, Andrews SP, Ashkenazi A, et al. Autophagy and Neurodegeneration: Pathogenic Mechanisms and Therapeutic Opportunities. Neuron 2017; 93: 1015-1034.

33. Xu Y, Li Y, Ma L, Xin G, Wei Z, Zeng Z, et al. D-Galactose induces premature senescence of lens epithelial cells by disturbing autophagy flux and mitochondrial functions. Toxicol Lett 2018; 289: 99-106.

34. Kim K Y, Park K I, Kim S H, Yu S N, Park S G, Kim YW, et al. Inhibition of Autophagy Promotes Salinomycin-Induced Apoptosis via Reactive Oxygen Species-Mediated PI3K/AKT/mTOR and ERK/p38 MAPK-Dependent Signaling in Human Prostate Cancer Cells. Int J Mol Sci 2017; 18: 1088-1100.

35. Gomez-Cambronero J, Kantonen S. A river runs through it: how autophagy, senescence, and phagocytosis could be linked to phospholipase D by Wnt signaling. J Leukoc Biol 2014; 96: 779-784.

36. Salminen A, Kaarniranta K. SIRT1: regulation of longevity via autophagy. Cell Signal 2009; 21: 1356-1360.

37. Peck B, Chen CY, Ho KK, Di Fruscia P, Myatt SS, Coombes RC, et al. SIRT inhibitors induce cell death and $\mathrm{p} 53$ acetylation through targeting both SIRT1 and SIRT2. Mol Cancer Ther 2010; 9: 844-855. 
38. Lee IH, Cao L, Mostoslavsky R, Lombard DB, Liu J, Bruns NE, et al. A role for the NAD-dependent deacetylase Sirt1 in the regulation of autophagy. Proceedings of the National Academy of Sciences of the United States of America 2008; 105: 3374-3379.

39. Lu Z, Miao Y, Muhammad I, Tian E, Hu W, Wang J, et al. JNK mediated colistin-induced autophagy and apoptosis via JNK-Bc12Bax signaling pathway and JNK-p53-ROS positive feedback loop in PC12 cells. Chem Biol Interact 2017; 277: 62-73.

40. Corpas R, Revilla S, Ursulet S, Castro M, Kaliman P, Petegnief V, et al. SIRT1 Overexpression in Mouse Hippocampus Induces Cognitive Enhancement Through Proteostatic and Neurotrophic Mechanisms. Mol Neurobiol 2016; 54: 5604-5619.
41. Nixon RA, Wegiel J, Kumar A, Yu WH, Peterhoff C, Cataldo A, et al. Extensive involvement of autophagy in Alzheimer disease: an immunoelectron microscopy study. J Neuropathol Exp Neurol 2005; 64: 113-122.

42. Wang F, Li H, Yan XG, Zhou ZW, Yi ZG, He ZX, et al. Alisertib induces cell cycle arrest and autophagy and suppresses epithelialto-mesenchymal transition involving PI3K/Akt/mTOR and sirtuin 1-mediated signaling pathways in human pancreatic cancer cells. Drug Des Devel Ther 2015; 9: 575-601.

Publisher's Note Springer Nature remains neutral with regard to jurisdictional claims in published maps and institutional affiliations. 